\title{
Recent Highlights: Onco Viral Exploitation of the SUMO System
}

\author{
Domenico Mattoscio ${ }^{1,2 \star}$, Alessandro Medda ${ }^{3}$ and Susanna Chiocca ${ }^{3 \star}$
}

\author{
${ }^{1}$ Department of Medical, Oral, and Biotechnology Science, University of Chieti-Pescara, Chieti, \\ Italy. \\ ${ }^{2}$ Center on Aging Science and Translational Medicine (CeSI-MeT) 'G. d’Annunzio', University of \\ Chieti-Pescara, Chieti, Italy. \\ ${ }^{3}$ Department of Experimental Oncology, IEO, European Institute of Oncology IRCCS, Milan, Italy. \\ *Correspondence: d.mattoscio@unich.it and susanna.chiocca@ieo.it
}

https://doi.org/10.21775/cimb.035.001

\begin{abstract}
Small ubiquitin-like modifier (SUMO)ylation is a crucial post-translational modification that controls functions of a wide collection of proteins and biological processes. Hence, given its pleiotropic role, viruses have developed many approaches to usurp SUMO conjugation to exploit the cellular host environment for their own benefit. Consistently, cancer cells also frequently impact on SUMO to force cellular transformation, underlining the importance of SUMO in health and diseases. Therefore, after a brief introduction to the multistep SUMOylation pathway, in this review we will focus our attention on several examples of strategies adopted by oncogenic viruses to hijack SUMOylation in order to promote infection, persistence and malignant transformation of host cells.
\end{abstract}

\section{Introduction}

The Small Ubiquitin-like Modifier (SUMO) proteins are involved in post translational modification (PTM) of target proteins (Matunis et al., 1996; Kamitani et al., 1997). The name SUMO comes from a structural similarity with ubiquitin and from the similar mechanism by which it is attached to target proteins (Mahajan et al., 1997). Indeed, both ubiquitination and SUMOylation are reversible processes catalysed by a cascade of enzymes, namely E1, E2 and E3 proteins (Gong et al., 1997; Mahajan et al., 1997; Johnson and Gupta, 2001).

\section{SUMO proteins}

The expression of SUMO proteins is conserved among eukaryotes. Lower eukaryotes have only one SUMO, while higher eukaryotes express three or more SUMO paralogues. In particular, in humans five different isoforms of SUMO are present, differing for response to physiological or stress conditions, tissue-specificity, and the ability to form SUMO chains [recently reviewed in Yang et al., (2017)]. SUMO1 is 101 amino acids protein found almost always conjugated to targets, and therefore often associated to physiological processes (Shen et al., 1996; Yang et al., 2017). SUMO2 consists of 95 amino acids and shares 95\% homology with SUMO3 (103 amino acids), differing for only three $\mathrm{N}$-terminal residues, and showing the same molecular functions, therefore often referred as SUMO2/3. They show only $45 \%$ homology with SUMO1 but they present a very similar tridimensional structure. SUMO2/3 are conjugated mostly under stress conditions and they are able to form chains (Mannen et al., 1996; Lapenta et al., 1997). SUMO4 seems to be expressed only in lymph nodes, kidneys and 
spleen. SUMO4 has not been well characterized yet and its role still needs to be elucidated. It is probably non-conjugated under physiological conditions and it has been associated to diabetes (Wang et al., 2006). Finally, the expression of a fifth SUMO isoform has been recently reported (Liang et al., 2016). SUMO5 seems to be a conserved 84 amino acids protein whose mRNA is expressed at high levels in testes and peripheral blood lymphocytes, and at lower levels also in placenta, lungs and liver. Conjugation with this novel SUMO variant facilitates the formation of Promyelocytic Leukaemia Nuclear Bodies (PML$\mathrm{NB}$ ), structures rich in SUMOylated proteins that regulate a variety of cell functions.

\section{The SUMO machinery}

The SUMOylation process is carried out in different steps (Fig. 3.1). Initially, SUMO is processed by a protease (belonging to the SENP family, as described in more details below), that generates the mature form consisting of a C-terminal diglycine (Hickey et al., 2012). This motif is required for the following step, in which the SUMO E1 enzyme activates SUMO. There is one only SUMO
E1 enzyme expressed in mammalian cells, a heterodimer composed by SUMO-activating enzyme subunit 1 (SAE1) and ubiquitin-like activating enzyme subunit 2 (SAE2/UBA2) (Desterro et al., 1999). SUMO is adenylated by the E1 complex in an ATP. $\mathrm{Mg}^{2+}$-dependent reaction and transferred to the catalytic Cys of the UBA2 subunit by an E1 SUMO thioester bond. Then, the unique SUMO E2 conjugating enzyme, ubiquitin-like conjugating 9 (UBC9), receives SUMO on a conserved catalytic cysteine, forming an E2 SUMO thioester complex (Tong et al., 1997; Duan et al., 2009). The E2 enzyme can attach SUMO to substrates, with the formation of an isopeptide bond between the carboxy-terminal carboxyl group of SUMO and a $\varepsilon$-amino group of the substrate acceptor Lys residue. UBC9 can be itself modified by different PTMs which increase or decrease its activity and localization, and confer substrate specificity (Knipscheer et al., 2008; Su et al., 2012). UBC9 can interact directly with some SUMO substrates but more often it needs the help of SUMO E3 enzymes, ligases that are able to give specificity to the targets (Sachdev et al., 2001; Tatham et al., 2005). Opposite to the unique E2, there are different SUMO

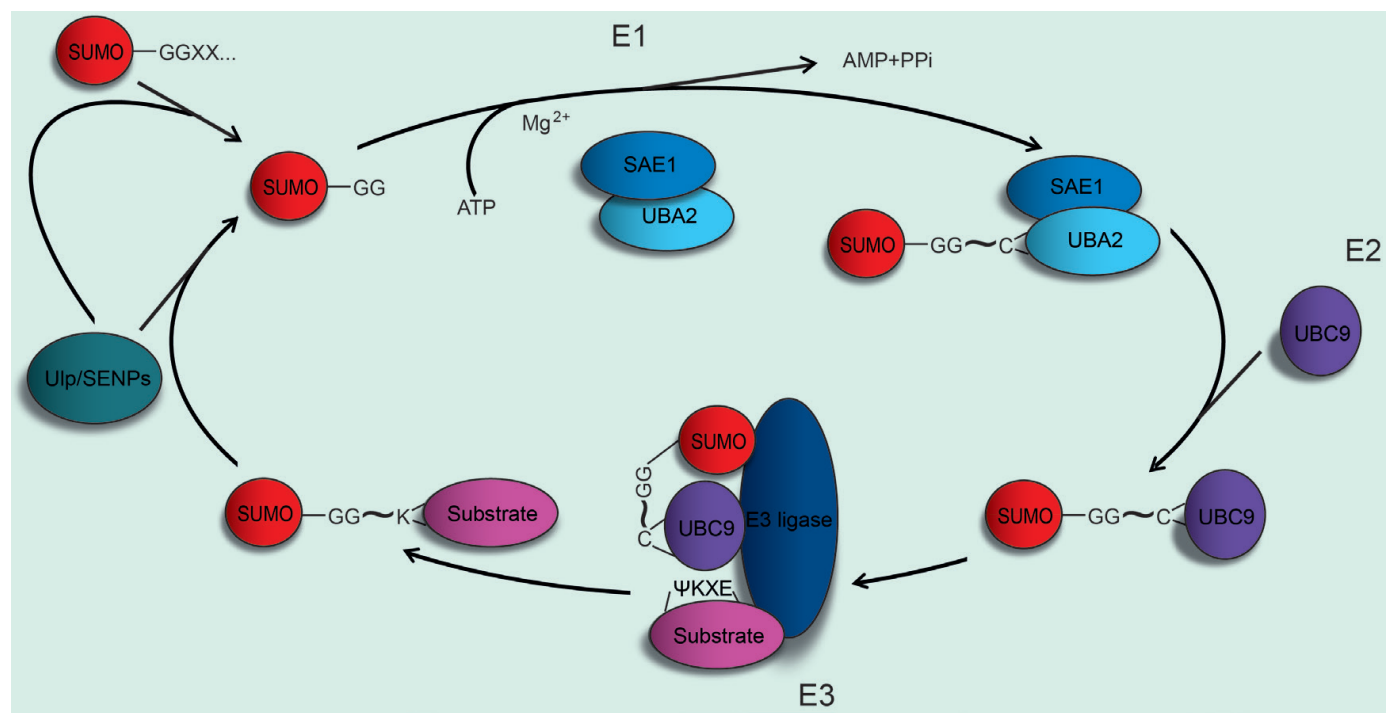

Figure 3.1 The SUMO conjugation system. Ulp1/SENP proteases catalyse cleavage of the C-terminal domain of SUMO proteins, exposing a diglycine motif. Processed SUMO is transferred to a cysteine of the heterodimeric E1 enzyme Uba2/SAE1. SUMO is then conjugated to a cysteine of UBC9, the E2 enzyme and attached to a lysine residue of the consensus motif on target proteins. The conjugation is often facilitated by an E3-ligase, which enforces the interactions among the involved components. SUMOylation is a reversible pathway where the UIp1/SENPs proteases dictate the de-SUMOylation process. 
E3 ligases, selective for SUMO1 or SUMO2/3 [described in Mattoscio and Chiocca (2015)]. Some E3 ligases act by orientating the E2 SUMO thioester in an optimal conformation for catalysis without directly contacting the substrate, while others facilitate the release of SUMO from E2 [reviewed in Wilkinson and Henley (2010)]. As mentioned before, SUMOylation is a reversible process governed by the action of two families of proteases that deconjugate SUMO from substrates. They include Ubl-specific proteases and sentrinspecific proteases (Ulps and SENPs, respectively) (Li and Hochstrasser, 2000).

\section{SENPS}

The human SENP family is composed of seven members: SENP1, SENP2, SENP3, SENP5, SENP6, SENP7 and SENP8, even if SENP8 is not specific for SUMO but acts on Nedd8, another ubiquitin-like protein (Hickey et al., 2012). SENPs are cysteine proteases with a papain-like folded catalytic domain and specific $\mathrm{N}$-terminal domains crucial for their own regulation and for substrate selection (Hay, 2007).

SENP proteases regulate both the level of processed SUMO and the rate of substrate modification by counterbalancing SUMO conjugation [recently reviewed in Kunz et al. (2018)]. In the maturation process they hydrolyse a peptide bond close to the C-terminus of SUMO precursors, eliminating the very $\mathrm{C}$-terminal amino acids from SUMO1, SUMO2 and SUMO3 and exposing two glycine residues. In SUMO1-3, the diGly motif is preceded by a glutamine $(\mathrm{Q})$ and threonine $(\mathrm{T})$, while SUMO4 exhibits a PTGG motif, in which the proline residue confers resistance to SENPmediated cleavage (Owerbach et al., 2005). In the deconjugation process, SENPs cleave an isopeptide bond that links SUMO moieties to the $\varepsilon$-amino group of lysine residues.

The mechanism by which SENP1 and SENP2 exert their functions has been described by X-ray crystallography. In vitro protease assays with the isolated catalytic domains demonstrate the processing activity of SENP1 and SENP2 on all three SUMO precursors (Reverter and Lima, 2006; Shen et al., 2006a). However, they exert differential activities towards distinct precursors. In particular, SENP2 is most active on SUMO2, then SUMO3 and SUMO1, while SENP1 prefers SUMO1.
Probably these differences are due to the amino acid sequences of the C-terminal tail. SENP5 has been found to have a marked preference for SUMO2 cleavage, while SENP6 and SENP7 are not able to process SUMO for maturation.

The SUMO system leaves a dilemma on how it is possible to achieve specificity on SUMOylation of a myriad of proteins with the small numbers of conjugating and deconjugating enzymes available. In some cases, specific biological processes are regulated by distinct deconjugation events. Though, in many cases, a single SENP may act on larger groups of SUMOylated proteins (Psakhye and Jentsch, 2012; Jentsch and Psakhye, 2013). Moreover, alternative splicing and PTMs of SENPs are important to determine their localization and their protease activity. To summarize, the special control of SENPs is a fundamental principle for deSUMOylation regulation (Kunz et al., 2018).

\section{The SUMO consensus motifs}

The main SUMO consensus motif existing in the primary structure of SUMOylated protein is $\psi \mathrm{KX}(\mathrm{D} / \mathrm{E})$, where $\psi$ is a large hydrophobic residue, $\mathrm{X}$ is any amino acid and $\mathrm{K}$ is the acceptor lysine. These residues directly bind UBC9 and are crucial for a stable interaction between the $\mathrm{E} 2$ enzyme and the substrate (Rodriguez et al., 2001). In addition to the canonical four amino acid SUMO consensus motifs, longer sequences that include both SUMO consensus motifs and additional elements have been identified in some SUMO substrates (Gareau and Lima, 2010). Among these, phosphorylationdependent SUMO motifs (PDSMs) and negatively charged amino acid-dependent SUMO motifs (NDSMs) are present. PDSMs present a SUMO consensus motif located adjacent to a phosphorylation site, $\psi \mathrm{KX}(\mathrm{D} / \mathrm{E}) \mathrm{XXSP}$. Phosphorylation increases SUMO conjugation levels because the phosphorylated Ser side chain interacts with a basic patch on the E2 surface, extending interactions with the E2 enzyme beyond recognition of the SUMO consensus motif. This mechanism is probably shared with proteins that contain NDSMs, which comprise negatively charged residues that are C-terminal to the SUMO consensus site in the place of the phosphorylation site of PDSMs, although NDSMs may interact with a different subset of Lys residues on the UBC9 surface (Yang et al., 2006; Mohideen et al., 2009). Recent studies 
revealed new motifs for SUMO conjugation, including inverted consensus motifs and motifs with an N-terminal hydrophobic cluster. These alternative motifs are probably important to give specificity to E2-substrate interactions through direct interaction with the E2 (Impens et al., 2014).

\section{SIM}

SUMO interacting motifs (SIMs) establish noncovalent hydrophobic interaction between SUMO and the target proteins. Canonical SIMs contain a core of hydrophobic residues that can be preceded or followed by negatively charged amino acids that take contact with hydrophobic groove on SUMO with following basic residues. This interaction is generally weak but can be increased by the binding of multiple SIMs to SUMO chains. Crucial hydrophobic and basic residues involved in SIM binding are conserved among the SUMO paralogues. However, the isoforms might differ in the placement of their hydrophobic groove suggesting that the arrangement of hydrophobic and acidic residues in SIMs might dictate their ability to bind specific SUMO isoforms (Hecker et al., 2006; Kerscher, 2007).

\section{STUbLs}

SUMO Targeted Ubiquitin Ligases (STUbLs) are Ubiquitin E3 enzymes able to recognize SUMOylated proteins and to interact with them through SIMs. They attach ubiquitin chains to SUMOylated proteins to target them for degradation by the proteasome. STUbLs constitute an important regulatory mechanism to control the levels of the SUMO conjugated form of a protein (Sriramachandran and Dohmen, 2014). The human RING Finger protein 4 (RNF4) is one of the best studied STUbL, containing at least three SIMs. These motifs mediate a similar non-covalent interaction with SUMO1 and SUMO2, with a preference for chains of a length of at least three SUMO moieties. RNF4 works as homodimer, in which the RING domains of both subunits take contact with a single ubiquitin-charged E2 (Sun et al., 2007).

\section{SUMO functions}

SUMOylation is involved in many different biological processes and can confer different properties to substrate proteins. A high number of known SUMOylation targets are nuclear proteins, involved in DNA repair, regulation of transcription and chromatin structure. Many important nuclear targets of signalling pathways can be SUMOylated. SUMOylation is important for subcellular localization of proteins, competes with other PTMs, and also participates to proteinprotein interaction. SUMOylation can also change the interaction between DNA and RNA, alter enzymatic activity and protein conformation, and modulate other modifications (recently reviewed in (Zhao, 2018). In the following paragraphs we will describe some paradigmatic example of how SUMOylation can impact on the activity of important selected targets.

\section{RanGAP}

RanGAP, the first protein shown to be SUMO modified, is important for nuclear import. Unmodified RanGAP is cytoplasmic, whereas SUMO-modified RanGAP is associated with the nuclear pore. SUMOylation of RanGAP increases its interaction with the SUMO E3 ligase Ran binding protein 2, a component of the nuclear pore complex. Localization of the RanBP2 SUMO E3 ligase at the nuclear pore could be important for a broad role for SUMO in regulation of nuclear trafficking (Matunis et al., 1996).

\section{Promyelocytic leukaemia protein}

Promyelocytic leukaemia protein (PML) is posttranslationally modified by SUMO and is localized in subnuclear structures named PML nuclear bodies, structures highly enriched in SUMOylated proteins. PML bodies host more than 150 proteins with a wide range of functions, such as DNA repair, stress response, senescence, anti-viral immunity, and tumour suppression. Notably, a variety of SUMO-modified proteins including transcription factors, chromatin modifiers, and proteins involved in genomic maintenance, are expressed in PML nuclear bodies together with SUMO E3 ligases and SUMO-specific proteases. SUMO-modified PML probably supports some protein-protein interactions important for assembly or stability of this subnuclear domain. Focused studies of cellular membrane-less structures suggest that proteins able to form inter-molecular multivalent interactions can constitute large oligomers and phase separate 
from the surrounding solution. These proteins can use their interaction domains or intrinsically disordered regions to recruit additional macromolecules even in high concentrations, maybe promoting certain biological processes (Shen et al., 2006b).

\section{SUMO modulation of chromosomes and chromatin}

SUMO deficiency drastically changes chromosome integrity and segregation. Indeed, SUMO enzymes are enriched at centrosomes, very important structures that support kinetochores for microtubules attachment during cell division (Lapenta et al., 1997). Topoisomerase II is recruited to centromeres on SUMOylation of its non-catalytic C-terminus, to uncoil intertwined DNA before anaphase and facilitate centromeric segregation. Centromeric histones and chromatin regulators are also regulated by SUMO conjugation. In particular, SUMOylated Orc2 recruits the histone demethylase KDM5A to demethylate $\mathrm{H} 3 \mathrm{~K} 4 \mathrm{me} 3$ into H3K4me2, enhancing non-coding RNA synthesis from the locus and subsequent heterochromatin maintenance. Moreover, Aurora B kinase deSUMOylation facilitates its localization to the spindle mid-zone, essential step during mitosis [recently reviewed in Zhao (2018)].

The SUMO pathway does not regulate only centromeric regions. For example, the heterochromatin assembly factor HP1 is SUMOylated to promote its association with RNA transcripts located at these regions. In addition, SENP7 activity is important for HP1 regulation in order to retain it at heterochromatin, even if the molecular details are still unclear (Maison et al., 2012). SUMOylation also affects chromatin modifiers such as histone deacetylase 1 (HDAC1), an essential epigenetic regulator of a conserved family of deacetylases frequently involved in cancer progression (Ropero and Esteller, 2007). Indeed, in non-tumourigenic cells, SUMOylation of HDAC1 by SUMO1 promoted by the overexpressed PIASy triggers its ubiquitination and degradation in a proteasome-dependent manner, thus reducing HDAC1 expression. Conversely, in breast cancer cell lines, HDAC1 is preferentially conjugated by SUMO2 that protects HDAC1 from ubiquitin conjugation and degradation. Therefore, SUMOylation significantly affects the expression and activity of an important chromatin modifier involved in breast cancer progression (Citro et al., 2013).

In addition, SUMO plays a fundamental role in DNA double-strand breaks (DSB), where SUMOylation enables broken DNA ends to move outside to prevent illegitimate repair of repetitive sequences. Similarly, SUMO promotes movement of target eroded telomeres and DSB to nuclear periphery, in a STUbLs-mediated mechanism that interacts with SUMOylated DNA repair proteins leading to their proteasomal degradation [recently reviewed in Garvin and Morris (2017)].

\section{SUMO in DNA damage}

SUMOylation is important in the DNA damage checkpoint pathway. In both yeast and human cells, SUMOylation of DNA damage proteins occurs in parallel with checkpoint mediated phosphorylation. Interestingly, changes in the checkpoint pathway can modify SUMOylation events: decreasing Ataxia telangiectasia and Rad3 related (ATR) checkpoint kinase increases protein SUMOylation. Moreover, Ataxia-telangiectasia mutated (ATM) checkpoint kinase is able to increase SENP2 transcription in particular contexts, but also ATM seems to promote SUMOylation in the absence of ATR. This phenomenon suggests a context-dependent crosstalk between these pathways (Munk et al., 2017).

As described, SUMOylation affects almost all cellular activities, resulting as a key pathway regulating cells physiology. However, conversely, it is evident that alterations in normal SUMOylation could completely subvert cell functions [reviewed in Flotho and Melchior (2013)]. Therefore, SUMO pathway components are frequently altered in human diseases such as cancer (Mattoscio and Chiocca, 2015; Seeler and Dejean, 2017), and often exploited by viruses. Interestingly, oncogenic viral infections can also increase metabolic and proangiogenic markers through expression of a very specific domain that also controls SUMO enzymes expression (Pozzebon et al., 2013). Viral exploitation of SUMOylation has been recently detailed in elegant reviews (Mattoscio et al., 2013; Lowrey et al., 2017; Wilson, 2017), to which readers can refer. In the following sections we will provide some classic examples on how oncogenic viruses 
impact SUMOylation to increase their ability to infect, persist, and transform host cells.

\section{Oncoviruses exploitation of the SUMO pathway}

Infection with oncogenic viruses is also involved in cancer pathogenesis, accounting for about $15 \%$ of total malignancies in 2012 (Plummer et al., 2016). Seven viruses are associated with human cancers, including hepatitis $B$ virus (HBV), hepatitis $C$ virus (HCV), high-risk human papillomaviruses (HPV), Epstein-Barr virus (EBV), Kaposi's sarcoma herpesvirus (KSHV, also known as human herpesvirus type $8 \mathrm{HHV}-8$ ), human T-cell leukaemia virus type 1 (HTLV-1), and the recently emerged Merkel cell polyomavirus (MCPyV) (Mesri et al., 2014; Spurgeon and Lambert, 2013).

\section{Hepatitis $B$ virus}

$\mathrm{HBV}$ is a partially double-stranded circular DNA virus belonging to the Hepadnaviridae family. Persistent infection with HBV is associated with several liver diseases such as hepatocellular carcinoma (HCC), the most common cancer of the liver [reviewed in Di Bisceglie (2009)]. HCC pathogenesis is a combination of both indirect effects as a consequence of the chronic inflammatory condition due to the persistent HBV presence in liver cells, and directly through viral proteins expression. In particular, HBV X antigen ( $\mathrm{HBx}$ ), a viral product that acts as transcriptional cofactor during viral replication, is also able to promote cellular transformation altering crucial cellular pathways involved in cell growth, DNA repair, apoptosis, and cell cycle progression [recently reviewed in Xie (2017)].

Notably, several of these modifications are mediated by exploitation of SUMO pathway by HBx. Indeed, in HBV infected cells, HBx promotes deSUMOyation and relocalization of the host transcription factor Sp110, usually conjugated to SUMO1 and expressed inside PML-NBs. The detachment of SUMO1 moiety and the resulting Sp110 differential distribution in infected cells increases viral DNA load, decreases apoptosis and increases viability of hepatocytes, and markedly affects expression levels of genes involved in type I interferon pathway, a common response mechanism to viral infections. Mechanistically, $\mathrm{HBx}$ may promote the formation of Sp110-SENP1-HBx complex able to catalyse SUMO1 removal from Sp110 and to translocate HBx to Sp110 gene promoters in order to reprogram host gene expression and to trigger viral proliferation (Sengupta et al., 2017). These findings highlight the importance of the SUMOylation and deSUMOylation switch in the infection lifecycle and tumorigenesis triggered by HBV.

In addition, $\mathrm{HBx}$ expression in mice and human cell lines prompts cell growth altering the SUMOylation status of E-cadherin, a membrane protein crucially involved in epithelial-mesenchymal transition (EMT). Opposite to SUMOylation of Sp110, HBx expression promotes SUMO1 and 2/3-conjugation to E-cadherin, leading to E-cadherin degradation, EMT-transition, loss of cell-to-cell contact, and overgrowth of hepatocytes (Ha et al., 2016). Notably, SUMO1, SUMO2/3, SAE1/2, UBC9, and SENP2 are differentially expressed in HCC and play key roles in HCC pathogenesis [(Liu et al., 2015), as recently reviewed in Tomasi and Ramani (2018)], further underlining the importance of SUMOylation in liver cells transformation. However, if these alterations are directly mediated by HBV proteins or are a consequence of cancer growth is still an unresolved issue and will not be further described in this review.

\section{Hepatitis $\mathrm{C}$ virus}

Together with HBV, HCV is another important aetiological agent of HCC (Di Bisceglie, 1995). $\mathrm{HCV}$ is an enveloped, single-stranded RNA virus belonging to the Flaviviridae family. Similarly to $\mathrm{HBV}, \mathrm{HCV}$ can promote HCC development as a consequence of the chronic inflammatory condition associated with its persistence in hepatocytes, or through direct effects mainly mediated by the viral core, non-structural proteins 3 (NS3), and NS5A, crucial players in viral replication and in alteration of the host gene expression landscape [reviewed in Irshad et al. (2017)]. In particular, NS5A affects cellular pathways involved in liver cell proliferation, apoptosis immune response, and DNA repair (Irshad et al., 2017), and requires SUMOylation to increase its stability in host cells and to promote $\mathrm{HCV}$ replication. Indeed, NS5A is SUMOylated in the context of HCV infection by both SUMO1 and SUMO2/3, perturbing ubiquitination occurring at the same target lysine, and suppressing NS5A 
proteasomal degradation. In addition to stability, SUMOylation also regulates the interaction of NS5A with NS5B occurring during replication complex formation (Lee et al., 2014), a key event for viral replication. Indeed, SUMO1 is overexpressed in infected Huh7.5 cells (Akil et al., 2016), and abrogation of SUMO conjugation by Ubc9 silencing markedly impairs HCV RNA replication (Lee et al., 2014; Akil et al., 2016), suggesting the importance of SUMOylation for HCV lifecycle in hepatocytes. However, on the contrary, a recent report showed that SUMO removal obtained by silencing of PIAS2 during HCV infection enhances stability and expression of NS3 and NS5A, and increases HCV replication, in a SUMO1-dependent manner (Guo et al., 2017). Reasons for these apparent discrepancies are currently unknown and future studies are therefore needed to better clarify whether HCV-mediated tumorigenesis benefits or is dampened by SUMO.

\section{Human papilloma virus}

High-risk HPV types $(16,18,31,33,35,39,45$, 51, 52, 56, 58, and 59) (Bouvard et al., 2009) are the aetiological agent of cervical cancer and are also associated with other anogenital malignancies, such as vulvar, vaginal, anal, and penile cancers, and with a significant proportion of oropharyngeal tumours [reviewed in (zur Hausen, 2009)]. HPVs are double-stranded DNA viruses that promote malignant transformation in chronically infected keratinocytes of epithelia mainly due to the viral oncoproteins E6 and E7 that, through degradation of tumour suppressors p53 and retinoblastoma $(\mathrm{pRb})$, modify fundamental cellular pathways involved in cell cycle, apoptosis, DNA repair, and senescence [reviewed in Tommasino (2014)]. In addition to E6 and E7, HPV infection in keratinocytes entails the concerted and sequential action of other early non-structural proteins E1, E2, E4 and E5, and viral capsid protein L1 and L2 [reviewed in Woodman et al. (2007)]. Most of these viral proteins exploit SUMOylation to subvert cellular pathways and promote viral persistence in the host.

E2 is a multifunctional regulatory protein that binds to viral DNA and interacts with cellular proteins to regulate viral gene expression, partitioning and replication, and to modify host transcriptome (reviewed in (McBride, 2013). HPV18 E2 is a substrate for mono SUMOylation in vitro, in an E. coli expression system, and in HeLa cells after overexpression of HPV16 E2, Ubc9, and either SUMO1, 2 , or 3, despite a preference for SUMO2/3. Notably, the defective E2 SUMO mutant shows defects in transcriptional ability, suggesting a crucial role for SUMOylation in mediating E2 activities during HPV-mediated transformation (Wu et al., 2008). In addition, SUMOylation at K292 increases E2 expression levels after exogenous overexpression of SUMO components and by endogenous elevation of SUMOylation obtained after heat shock, due to a SUMO-mediated inhibition of E2 ubiquitination and degradation (Wu et al., 2009). Combining these results with the observation that SUMO2/3 is progressively up-regulated during keratinocytes differentiation (Deyrieux et al., 2007), the emerging scenario depicts that the increased SUMO2/3 expression in suprabasal layer of epithelium stabilizes E2, increases E2 concentration and activity, and promotes viral production (Wu et al., 2007).

The E6 and E7 viral oncoproteins drive malignant transformation mostly due to the degradation of p53 and pRb, respectively. However, in addition to these two well characterized pathways, a number of other cellular proteins are affected by E6 and E7 during viral infection and transformation [reviewed in Moody and Laimins (2010)]. Among these, clever strategies are adopted by HPV oncoproteins to hijack SUMO during infection and tumorigenesis. HPV16 E6 and E7 overexpression in the natural host of the virus, primary human keratinocytes, significantly increases the accumulation of UBC9 and SUMO1-conjugated species (Mattoscio et al., 2017). Notably, similar results were also found in human samples during the natural evolution of cervical and oropharyngeal cancer (Mattoscio et al., 2015, 2017), underlining the importance of SUMO alterations during HPV transformation. Mechanistically, HPV16 E6/E7 prevents the autophagy-dependent UBC9 degradation obstructing the final step of autophagic pathway in E6/p53-dependent manner (Mattoscio et al., 2017, 2018). The resulting increased UBC9 level confers apoptosis resistance to the infected keratinocyte (Mattoscio et al., 2017), thus extending HPV persistence in the host and triggering cellular transformation. However, this E6-mediated UBC9 accumulation seems to be a cell-specific mechanism dependent on the cellular background of analysed cells. Indeed, E6 overexpression in 
immortalized, p53 defective, cell lines drive UBC9 degradation through proteasomal-dependent pathway (Heaton et al., 2011) while in primary, p53 competent cells, E6 triggers UBC9 accumulation following autophagy defects. These and other results (Boggio et al., 2004, 2007) highlight a dual way to control UBC9 levels, further underlining the fundamental role of the SUMO E2 enzyme in cellular physiology.

In addition to impact on UBC9 expression, E6 also co-opts SUMOylation to re-direct activities of cellular transcription factors. Indeed, the transcriptional co-activator hADA3 (human alteration/deficiency in activation3), is down-regulated by HPV16 E6 in cervical cancer cells. In contrast to HPV E2 that exploits SUMO-conjugation to protect its own ubiquitin-dependent proteasomal degradation, HPV16 E6 triggers SUMOylation to induce ubiquitin attachment and hADA3 degradation. SUMO-dependent hADA3 deprivation encourages cell proliferation, migration, and anchorage independent growth of cervical cancer $\mathrm{SiHa}$ cells, pointing to the important role of SUMOylation in malignant transformation (Chand et al., 2014).

Similarly, in addition to contributing to UBC9 overexpression, E7 also usurps SUMOylation to regulate levels and activity of a transcription factor crucially involved in cell cycle progression, cell proliferation, and DNA damage response, Forkhead box M1b (FoxM1b). FoxM1 de-regulation occurs in a variety of malignancies [reviewed in Myatt and Lam (2007)], where its activity and expression are frequently modified by PTMs [reviewed in van der Horst and Burgering (2007)], including SUMOylation. Indeed, in vivo SUMOylation assays in HEK293T cells show that FoxM1 could be modified by all three SUMO paralogues after physical interaction with UBC9 and PIAS1. Similar results were obtained also in MCF-7 cells (Myatt et al., 2014). After SUMO conjugation, FoxM1 is rapidly degraded and re-localizes from nucleus to cytoplasm, suggestive of a negative regulatory loop mediated by SUMOylation to turn off its transcriptional activity (Myatt et al., 2014; Jaiswal et al., 2015). Notably, HPV16 E7 interferes with SUMO loading on FoxM1 by inhibiting its association with UBC9, in turn reducing FoxM1 SUMOylation and protecting it from re-localization and degradation (Jaiswal et al., 2015). SUMOylated FoxM1 increases cell proliferation and delays mitotic progression (Myatt et al., 2014), indicating the importance of the E7-mediated SUMO manipulation in the context of HPV-mediated cellular transformation.

Finally, SUMOylation of the late structural capsid protein L2 also plays crucial role in HPV infectivity and cellular transformation. Indeed, modification with SUMO2/3 increases L2 halflife and inhibits interaction with the other capsid protein $\mathrm{L} 1$, suggesting that capsid assembly could be modulated by SUMOylation during HPV infection (Marusic et al., 2010).

\section{Epstein-Barr virus}

EBV was the first virus clearly connected with human malignancies, since it was isolated in 1964 in cultured lymphoblasts from Burkitt's lymphoma cells (Epstein et al., 1964). Since then, EBV infection was also consistently associated with a number of other malignancies such as nasopharyngeal cancer, Hodgkin's and non-Hodgkin's lymphomas, and a subset of gastric cancers [reviewed in Thompson and Kurzrock (2004)]. EBV is a double-stranded DNA Herpesvirus that could establish latent and lytic infection in lymphoblastoid cells, characterized by restricted viral gene expression and life-long persistence, and with virions production, respectively, in lymphocytes and epithelial cells. Several proteins are involved and expressed in lytic reactivation, to promote cell proliferation, virus production, and oncogenesis [reviewed in Tsurumi et al. (2005)]. Among them, the transcriptional activator Zta could be modified by both SUMO1 and SUMO2/3 (Adamson and Kenney, 2001; Hagemeier et al., 2010) at K12. SUMOylated Zta associates with and carries HDAC3 on its targeted promoters which, in this way, acetylates and exerts an inhibitory activity at Zta-responsive genes (Murata et al., 2010). Consistently with the role of SUMOylation in mediating repression of $Z$ ta, the SUMO defective mutant increases gene expression and re-activation of latent EBV. Notably, the SUMO-mediated repression of Zta in vivo could be reverted by both the viral encoded EBV kinase (EBV-PK) and RanBPM during infection, reducing Zta SUMO conjugation, promoting transcription of $\mathrm{Zta}$ genes and replication of the viral genome (Hagemeier et al., 2010; Yang, Y.C. et al., 2015). SUMOylation and activity of Zta are also 
finely regulated by interaction of SUMOylated Zta with SIM motifs of the EBV protein kinase BGLF4. After SUMO-mediated Zta-BGLF4 interaction, the kinase activity of BGLF4 abolishes Zta SUMOylation, activating virus production ( $\mathrm{Li}$ et al., 2012).

Similar to Zta, the activity of the other EBV protein involved in lytic reactivation, Rta (Tsurumi et al., 2005), is crucially regulated by SUMO. Yeasttwo-hybrid screen identifies UBC9 and PIAS1 as binding partners of Rta, that is SUMOylated both in vitro and in vivo during the early stages after lytic induction of EBV infection. However, contrary to Zta, SUMO1 conjugation increases the transcriptional ability of Rta, suggesting a crucial role for SUMOylation for EBV lytic reactivation. Indeed, since Rta mediates Zta transcription (Adamson and Kenney, 2001), SUMO could both serve initially as activator of lytic phase by conjugating to Rta and promoting transcription of genes such as Zta, and then as modulator of EBV reactivation through SUMOylation-mediated Zta repression triggered by viral EBV-PK and cellular RanBPM.

Latent membrane protein 1 (LMP1) is primarily involved in EBV oncogenesis due to its ability to mimic CD40 receptor and to constitutively transduce growth signals that trigger tumorigenesis in infected cells (Gires et al., 1997). LMP1 physically interacts with UBC9 to increase protein SUMOylation in latent infected cells (Bentz et al., 2011). Among SUMOylated proteins, LMP1-UBC9 interaction promotes SUMO conjugation of Interferon Regulatory Factor 7 (IRF7) to promote its nuclear localization and increases its stability in EBV infected cells. However, despite nuclear accumulation, SUMOylation inhibits IRF7 association with chromatin, thus reducing its transcriptional activity and the ability to induce innate immune response (Bentz et al., 2012). Moreover, LMP1 aids to preserve viral latency (Adler et al., 2002) and SUMOylation plays pivotal roles also in EBV lytic reactivation. Indeed, LMP1 triggers SUMOylation of the transcriptional repressor KRAB-associated protein-1 (KAP1). In EBV-transformed lymphoblastoid cell line, SUMOylated KAP1 associates with viral EBV lytic promoters OriLyt, ZTA and RTA, promoting the transcriptional repression that contributes to the maintenance of viral latency (Bentz et al., 2015).
In addition to LMP1, a recently reported genome-wide screening identifies other EBV proteins having global effects on host SUMOylation. In particular, overexpression of the transcriptional activator BRLF1 consistently decreased levels of both SUMO1 and SUMO2 conjugated proteins in transfected 293T and HeLa cells, while six EBV proteins up-regulated SUMOylation. Among them, expression of SM, an mRNA binding protein, increases levels of SUMO1 and to less extent SUMO2 conjugated proteins. This effect is due to the ability of SM to interact and bind UBC9 and SUMO, thus acting as an E3 ligase that promotes SUMO conjugation of cellular proteins such as p53. Consistently, SM depletion in AGS-EBV infected cells reduces global SUMOylation levels, suggesting the ability of SM to affect SUMOylation during viral lytic infection (De La Cruz-Herrera et al., 2018).

In addition to proteins, EBV also encodes a variety of microRNAs (miRNAs) during viral infection and oncogenesis. Bioinformatic analysis based on miRNA target prediction identified 575 proteins of the SUMO interactome that could be potentially targeted and modulated by EBV miRNAs and a set of 14 predicted 3' UTR were also experimentally validated in luciferase reporter assays. SUMO proteins targeted by EBV miRNAs are mainly involved in cancer-related functions such as proliferation, apoptosis, growth signalling, and intercellular communication, suggesting that miRNAs play fundamental roles during EBV carcinogenesis (Callegari et al., 2014). Accordingly, the EBV-encoded miR-BHRF-1 promotes accumulation of SUMO2/3 conjugated proteins during lytic infection due to down-regulation of RNF4 ( $\mathrm{Li}$ et al., 2017).

\section{Kaposi's sarcoma-associated herpesvirus}

KSHV is responsible for Kaposi's sarcoma, a malignancy commonly occurring in AIDS patients. In addition to Kaposi's sarcoma, KSHV has been detected in primary effusion lymphoma and in multicentric Castleman's disease [reviewed in Ganem (2006) ]. KSHV is a double-stranded DNA herpes virus that primarily infects endothelial and B cells that frequently exploits SUMOylation to promote its replication [recently reviewed in Chang and Kung (2014)]. Similar to EBV, KSHV infection 
cycle can be divided in lytic and latent phases. Viral reactivation can be triggered by a number of specific environmental stimuli and by the viral protein K-Rta. The lytic phase is characterized by a short period where viral genes are expressed, where during the latency there is the expression of a limited number of viral genes without production of viral particles (Aneja and Yuan, 2017). One of the most abundantly genes expressed during latent phase, the latency-associated nuclear antigen (LANA) protein, is a crucial regulator of dormant infections, viral reactivation and cellular transformation [reviewed in Uppal et al. (2014)]. To exert its functions, LANA extensively exploits SUMOylation machinery. Indeed, LANA contains a SIM to allow interaction with SUMO2 modified proteins such as KAP1 which is in turn recruited to specific chromatin sites to silence viral gene expression (Cai et al., 2013). Therefore, the LANA SIM motif plays a fundamental role in KSHV latency, even if data point to a direct binding of LANA with KAP1 independently of SIM (Sun et al., 2014). Furthermore, LANA is SUMOylated itself and its expression levels in KSHV infected SLK cells are regulated by a finely tuned deSUMOylation activity mediated by SENP6. Chromatin immunoprecipitation sequencing experiments identified that LANA binds SENP6 promoter, with subsequent repression of SENP6 expression. Given that SENP6 protease removes SUMO moieties from LANA to decrease its expression and to promote viral gene expression, these results suggest that LANA inhibits SENP6 to regulate its own SUMOylation and expression levels in infected cells, and to maintain KHSV latency (Lin et al., 2017).

KHSV encodes two additional transcription factors, K-bZIP (KSHV basic leucine-zipper) and K-Rta that are crucially regulated by SUMO. K-bZIP is an early lytic gene rapidly expressed after acute infection or during reactivation from latency (Lin et al., 1999), that acts as transcriptional repressor through inhibition of the viral transactivator K-Rta (Izumiya et al., 2003). Similar to LANA, also K-bZIP needs SUMOylation to increase its activity, since expression of the SUMO specific protease SENP1 attenuates transcriptional repression of K-Rta. K-bZIP could be conjugated by both SUMO1 and SUMO2/3 and requires interaction with UBC9 at viral promoters to mediate its repression activity (Izumiya et al., 2005). Consistently, ChIP-seq studies revealed deposition of SUMO2/3 throughout KSHV genome after viral reactivation, mirrored by decreased expression of KHSV genes (Yang, W.S. et al., 2015), suggesting that SUMOylation may be involved in chromatin remodelling during viral reactivation. Notably, K-bZIP also shows SUMO E3 ligase activity with specificity towards SUMO2/3 that catalyses SUMOylation of interacting partners such as $\mathrm{p} 53$ and $\mathrm{pRb}$ (Chang et al., 2010), and deposition of SUMO2/3 in chromatin locus enriched for SUMO2/3 (Yang, W.S. et al., 2015). Indeed, experimental KHSV reactivation in infected B lymphoma cell line is complemented by a specific increase of SUMO2/3 conjugation and inactivation of promoter regions of genes involved in immune response such as IRF-1, IRF-2, and IRF-7 (Chang et al., 2013). Collectively, these results suggest that SUMOylated K-bZIP interacts with UBC9, mediates SUMO2/3 modification of viral and cellular chromatin through its E3 SUMO ligase activity and shut-off of KHSV gene expression, and dampens the host immune activation, thus contributing to hide the virus from host responses during viral reactivation. Therefore, KHSV could regulate gene expression and viral replication manipulating SUMOylation. Indeed, modulation of global SUMO conjugation quickly occurs after induction of K-bZIP and K-Rta expression in chronically EBV-infected TRE $\times$ BCBL-1 $\mathrm{K}$ - Rta cell lines and is accompanied by modulation of viral gene expression (Wang et al., 2017). While K-bZIP promotes accumulation of SUMO-conjugated proteins, the viral activator K-Rta decreases global SUMOylation through its SUMO-targeting E3 ubiquitin ligase (STUbL)-like activity. STUbL proteins contain SIMs to interact with SUMO to ubiquitylate their targets (Perry et al., 2008). Indeed, K-Rta contains SIM domains able to bind SUMO moiety and to catalyse attachment of ubiquitin and proteasome-dependent degradation on targeted SUMOylated proteins. Among them, K-Rta promotes degradation of viral proteins like K-bZIP, and cellular proteins such as PML in order to create a conducive environment for viral replication (Izumiya et al., 2013). Therefore, KHSV expresses two different early genes acting as SUMO E3 ligases (K-bZIP) or STUbL (K-Rta) that differently affect SUMOylation status of infected cells in diverse phases of viral infection 
cycle. These examples are explanatory of how the dynamic and reversible alteration of SUMO conjugation represents a convenient strategy that oncogenic viruses exploit to alter and adapt host environment for viral purposes.

\section{Human T-cell leukaemia virus type 1}

HTLV-1 is the aetiological agent of adult T-cell leukaemia (Poiesz et al., 1980). Transforming ability of HTLV-1 mainly relies on the oncoviral protein Tax, a transcriptional activator able to initiate T-cell proliferation ad differentiation (Jeang et al., 2004). Activation of the NF-kB pathway, a crucial step towards transformation of a T-cell in a leukaemic cell, is finely regulated by concerted SUMO/ubiquitin conjugation steps that specifically shuttle Tax between cytoplasm and nuclear bodies. In particular, SUMOylated Tax is conjugated by ubiquitin through the STUbLs-mediated activity of RNF4 (Fryrear et al., 2012) and migrates to cytoplasm to allow interaction with the regulatory subunits of IkB kinase, NEMO, and the subsequent relocation of the Nf-KB subunit RelA to the nucleus. Then, deubiquitinated Tax translocates to nuclear bodies where it is SUMOylated on the same lysine residue by the resident UBC9 and SUMO, associates with RelA and NEMO, and starts the transcription of Tax-responsive genes mediated by NF-kB (Lamsoul et al., 2005; Nasr et al., 2006; Kfoury et al., 2011)

\section{Merkel cell polyomavirus}

$\mathrm{MCPyV}$ is the most recently emerged oncovirus, since it has been detected in about $80 \%$ of Merkel cell carcinoma, a neuroendocrine disorder of the skin frequently found in immune depressed patients [recently reviewed in (DeCaprio, 2017)]. MCPyV transforming ability mainly resides on the expression of Large $\mathrm{T}$ antigen proteins (Large- LT, and Short-ST) (Houben et al., 2010), even if molecular details driving MCPyV-mediated cell transformation are still not fully elucidated, although a role for ST is emerging (Shuda et al., 2011). Similarly, a role for viral exploitation of the SUMO pathway during Merkel cell carcinoma has not yet been investigated, even if a recent report shows that MCPyV replication depends on PML-NBs, suggesting a possible involvement of SUMOylation in regulating $\mathrm{MCPyV}$ transformation (Neumann et al., 2016).

\section{Conclusions}

Post-translational modification by SUMO plays central roles during oncogenic viral infections. SUMOylation is a physiological pathway regulating proteins activity, altering localization, interaction with DNA and other proteins [reviewed in Wilkinson and Henley (2010)]. At cellular levels, SUMOylation regulates processes such as cell division, DNA replication and repair, cell signalling, chromatin remodelling, apoptosis and proliferation [reviewed in Wilson (2009)]. Since the wide impact on cell physiology, alteration of SUMOylation is a convenient way that oncoviruses frequently exploit to mediate persistence in the host. Indeed, with the exception of the recently discovered $\mathrm{MCPyV}$, human oncovirus extensively manipulate SUMO to modify both viral and cellular proteins. Specifically, viral oncoproteins from HCV (NS5A), KHSV (LANA), and HTLV-1 (Tax), as well as viral structural and transcription factors such as HPV E2 and L2, EBV Zta and Rta, KHSV K-bZIP and K-Rta are all modified by one or more SUMO paralogues to alter their localization, transcriptional ability, protein-protein, protein-DNA interaction, and stability. Also, proteins from oncogenic viruses could act as specific SUMO E3 ligase to catalyse the addition of SUMO moiety to cellular target (K-bIZP) or as STUbL to clear SUMOylated proteins through the ubiquitin-mediated proteasomal degradation (K-Rta). Notably, these two apparently contrasting activities are mediated by the same oncovirus (KHSV) in different steps of viral infections, suggesting the importance of SUMOylation to quickly and completely revert cellular activities for virus purposes. Furthermore, several oncoviruses proteins, such as $\mathrm{HBx}, \mathrm{E} 6, \mathrm{E} 7$, and LMP1, mediate SUMOylation of specific transcription factors to trigger or dampen expression of genes that finally promote virus infectivity and oncogenesis. Similarly, viral proteins and miRNAs could also globally affect SUMOylation in infected cells, both increasing (E6/E7, LMP1, SM, miRBHRF1-1) or decreasing (BRLF1) SUMOylation of specific SUMO paralogues. Strikingly, the ability of altering global SUMOylation could also be contingent on the manipulation of the sole SUMO E2-conjugating enzyme, UBC9, whose alteration could completely revert host functions to advantage virus endurance, as exemplified by HPV E6. Collectively, findings summarized here clearly 
suggest that SUMOylation is a pivotal pathway during infection and transformation triggered by oncoviruses. Therefore, strategies aimed at interfering with viral manipulation of SUMO components could be beneficial in the attempt to reduce cancer burden arising from viral infection.

\section{Acknowledgements}

Work in the S.C. laboratory related to the topics discussed in this review is supported by Associazione Italiana per la Ricerca sul Cancro (AIRC). D.M. is a recipient of the Fondazione Umberto Veronesi (FUV) fellowship.

\section{References}

Adamson, A.L., and Kenney, S. (2001). Epstein-barr virus immediate-early protein BZLF1 is SUMO-1 modified and disrupts promyelocytic leukemia bodies. J. Virol. 75, 2388-2399. https://doi.org/10.1128/JVI.75.5.23882399.2001.

Adler, B., Schaadt, E., Kempkes, B., Zimber-Strobl, U., Baier, B., and Bornkamm, G.W. (2002). Control of EpsteinBarr virus reactivation by activated CD40 and viral latent membrane protein 1. Proc. Natl. Acad. Sci. U.S.A. 99, 437-442. https://doi.org/10.1073/pnas.221439999.

Akil, A., Wedeh, G., Zahid Mustafa, M., and GassamaDiagne, A. (2016). SUMO1 depletion prevents lipid droplet accumulation and HCV replication. Arch. Virol. 161, 141-148. https://doi.org/10.1007/s00705-0152628-3.

Aneja, K.K., and Yuan, Y. (2017). Reactivation and lytic replication of Kaposi's sarcoma-associated herpesvirus: an update. Front. Microbiol. 8, 613. https://doi. org/10.3389/fmicb.2017.00613.

Bentz, G.L., Whitehurst, C.B., and Pagano, J.S. (2011) Epstein-Barr virus latent membrane protein 1 (LMP1) C-terminal-activating region 3 contributes to LMP1mediated cellular migration via its interaction with Ubc9. J. Virol. 85, 10144-10153. https://doi.org/10.1128/ JVI.05035-11.

Bentz, G.L., Shackelford, J., and Pagano, J.S. (2012). Epstein-Barr virus latent membrane protein 1 regulates the function of interferon regulatory factor 7 by inducing its sumoylation. J. Virol. 86, 12251-12261. https://doi. org/10.1128/JVI.01407-12.

Bentz, G.L., Moss, C.R., Whitehurst, C.B., Moody, C.A., and Pagano, J.S. (2015). LMP1-induced sumoylation influences the maintenance of Epstein-Barr virus latency through KAP1. J. Virol. 89, 7465-7477. https://doi. org/10.1128/JVI.00711-15.

Boggio, R., Colombo, R., Hay, R.T., Draetta, G.F., and Chiocca, S. (2004). A mechanism for inhibiting the SUMO pathway. Mol. Cell 16, 549-561.

Boggio, R., Passafaro, A., and Chiocca, S. (2007). Targeting SUMO E1 to ubiquitin ligases: a viral strategy to counteract sumoylation. J. Biol. Chem. 282, 1537615382.

Bouvard, V., Baan, R., Straif, K., Grosse, Y., Secretan, B., El Ghissassi, F., Benbrahim-Tallaa, L., Guha, N., Freeman,
C., Galichet, L., et al. (2009). A review of human carcinogens - Part B: biological agents. Lancet Oncol. 10, 321-322.

Cai, Q., Cai, S., Zhu, C., Verma, S.C., Choi, J.Y., and Robertson, E.S. (2013). A unique SUMO-2-interacting motif within LANA is essential for KSHV latency. PLOS Pathog. 9, e1003750. https://doi.org/10.1371/journal. ppat.1003750.

Callegari, S., Gastaldello, S., Faridani, O.R., and Masucci, M.G. (2014). Epstein-Barr virus encoded microRNAs target SUMO-regulated cellular functions. FEBS J. 281, 4935-4950. https://doi.org/10.1111/febs.13040.

Chand, V.,John, R.,Jaiswal, N.,Johar, S.S., and Nag,A. (2014). High-risk HPV16E6 stimulates hADA3 degradation by enhancing its SUMOylation. Carcinogenesis 35, 1830-1839. https://doi.org/10.1093/carcin/bgu104.

Chang, P.C., and Kung, H.J. (2014). SUMO and KSHV replication. Cancers 6, 1905-1924. https://doi. org/10.3390/cancers6041905.

Chang, P.C., Izumiya, Y., Wu, C.Y., Fitzgerald, L.D., Campbell, M., Ellison, T.J., Lam, K.S., Luciw, P.A., and Kung, H.J. (2010). Kaposi's sarcoma-associated herpesvirus (KSHV) encodes a SUMO E3 ligase that is SIM-dependent and SUMO-2/3-specific. J. Biol. Chem. 285, 5266-5273. https://doi.org/10.1074/jbc. M109.088088.

Chang, P.C., Cheng, C.Y., Campbell, M., Yang, Y.C., Hsu, H.W., Chang, T.Y., Chu, C.H., Lee, Y.W., Hung, C.L., Lai, S.M., et al. (2013). The chromatin modification by SUMO-2/3 but not SUMO-1 prevents the epigenetic activation of key immune-related genes during Kaposi's sarcoma associated herpesvirus reactivation. BMC Genomics 14, 824. https://doi.org/10.1186/14712164-14-824.

Citro, S., Jaffray, E., Hay, R.T., Seiser, C., and Chiocca, S. (2013). A role for paralog-specific sumoylation in histone deacetylase 1 stability. J. Mol. Cell Biol. 5, 416-427. https://doi.org/10.1093/jmcb/mjt032.

De La Cruz-Herrera, C.F., Shire, K., Siddiqi, U.Z., and Frappier, L. (2018). A genome-wide screen of EpsteinBarr virus proteins that modulate host SUMOylation identifies a SUMO E3 ligase conserved in herpesviruses. PLOS Pathog. 14, e1007176. https://doi.org/10.1371/ journal.ppat.1007176.

DeCaprio, J.A. (2017). Merkel cell polyomavirus and Merkel cell carcinoma. Philos. Trans. R. Soc. Lond. B Biol. Sci. 372. pii: 20160276. https://doi.org/10.1098/ rstb.2016.0276.

Desterro, J.M., Rodriguez, M.S., Kemp, G.D., and Hay, R.T. (1999). Identification of the enzyme required for activation of the small ubiquitin-like protein SUMO-1. J. Biol. Chem. 274, 10618-10624.

Deyrieux, A.F., Rosas-Acosta, G., Ozbun, M.A., and Wilson, V.G. (2007). Sumoylation dynamics during keratinocyte differentiation. Journal of cell science 120, 125-136. https://doi.org/10.1242/jcs.03317.

Di Bisceglie, A.M. (1995). Hepatitis C and hepatocellular carcinoma. Semin. Liver Dis. 15, 64-69. https://doi. org/10.1055/s-2007-1007263.

Di Bisceglie, A.M. (2009). Hepatitis B and hepatocellular carcinoma. Hepatology 49, S56-60. https://doi. org/10.1002/hep.22962. 
Duan, X., Trent, J.O., and Ye, H. (2009). Targeting the SUMO E2 conjugating enzyme Ubc9 interaction for anti-cancer drug design. Anticancer. Agents Med. Chem. 9, 51-54.

Epstein, M.A., Achong, B.G., and Barr, Y.M. (1964). Virus particles in cultured lymphoblasts from Burkitt's lymphoma. Lancet 1, 702-703.

Flotho, A., and Melchior, F. (2013). Sumoylation: a regulatory protein modification in health and disease. Annu. Rev. Biochem. 82, 357-385. https://doi. org/10.1146/annurev-biochem-061909-093311.

Fryrear, K.A., Guo, X., Kerscher, O., and Semmes, O.J. (2012). The Sumo-targeted ubiquitin ligase RNF4 regulates the localization and function of the HTLV-1 oncoprotein Tax. Blood 119, 1173-1181. https://doi. org/10.1182/blood-2011-06-358564.

Ganem, D. (2006). KSHV infection and the pathogenesis of Kaposi's sarcoma.Annu. Rev. Pathol. 1,273-296.https:// doi.org/10.1146/annurev.pathol.1.110304.100133.

Gareau, J.R., and Lima, C.D. (2010). The SUMO pathway: emerging mechanisms that shape specificity, conjugation and recognition. Nat. Rev. Mol. Cell Biol. 11, 861-871. https://doi.org/10.1038/nrm3011.

Garvin, A.J., and Morris, J.R. (2017). SUMO, a small, but powerful, regulator of double-strand break repair. Philos. Trans. R. Soc. Lond., B, Biol. Sci. 372, 20160281.

Gires, O., Zimber-Strobl, U., Gonnella, R., Ueffing, M., Marschall, G., Zeidler, R., Pich, D., and Hammerschmidt, W. (1997). Latent membrane protein 1 of Epstein-Barr virus mimics a constitutively active receptor molecule. EMBO J. 16, 6131-6140. https://doi.org/10.1093/ emboj/16.20.6131.

Gong, L., Kamitani, T., Fujise, K., Caskey, L.S., and Yeh, E.T. (1997). Preferential interaction of sentrin with a ubiquitin-conjugating enzyme, Ubc9. J. Biol. Chem. 272, 28198-28201.

Guo, J., Chen, D., Gao, X., Hu, X., Zhou, Y., Wu, C., Wang, Y., Chen, J., Pei, R., and Chen, X. (2017). Protein inhibitor of activated STAT2 restricts HCV replication by modulating viral proteins degradation. Viruses 9, E285.

Ha, H.L., Kwon, T., Bak, I.S., Erikson, R.L., Kim, B.Y., and Yu, D.Y. (2016). IGF-II induced by hepatitis B virus $\mathrm{X}$ protein regulates EMT via SUMO mediated loss of E-cadherin in mice. Oncotarget 7, 56944-56957. https://doi.org/10.18632/oncotarget.10922.

Hagemeier, S.R., Dickerson, S.J., Meng, Q. Yu, X., Mertz, J.E., and Kenney, S.C. (2010). Sumoylation of the Epstein-Barr virus BZLF1 protein inhibits its transcriptional activity and is regulated by the virusencoded protein kinase. J. Virol. 84, 4383-4394. https:// doi.org/10.1128/JVI.02369-09.

Hay, R.T. (2007). SUMO-specific proteases: a twist in the tail. Trends Cell Biol. 17, 370-376.

Heaton, P.R., Deyrieux, A.F., Bian, X.L., and Wilson, V.G. (2011). HPV E6 proteins target Ubc9, the SUMO conjugating enzyme. Virus Res. 158, 199-208. https:// doi.org/10.1016/j.virusres.2011.04.001.

Hecker, C.M., Rabiller, M., Haglund, K., Bayer, P., and Dikic, I. (2006). Specification of SUMO1- and SUMO2interacting motifs. J. Biol. Chem. 281, 16117-16127.

Hickey, C.M., Wilson, N.R., and Hochstrasser, M. (2012). Function and regulation of SUMO proteases. Nat. Rev.
Mol. Cell Biol. 13, 755-766. https://doi.org/10.1038/ nrm3478.

Houben, R., Shuda, M., Weinkam, R., Schrama, D., Feng, H., Chang, Y., Moore, P.S., and Becker, J.C. (2010). Merkel cell polyomavirus-infected Merkel cell carcinoma cells require expression of viral T antigens. J. Virol. 84, 7064-7072. https://doi.org/10.1128/JVI.02400-09.

Impens, F., Radoshevich, L., Cossart, P., and Ribet, D. (2014). Mapping of SUMO sites and analysis of SUMOylation changes induced by external stimuli. Proc. Natl. Acad. Sci. U.S.A. 111, 12432-12437. https:// doi.org/10.1073/pnas.1413825111.

Irshad, M., Gupta, P., and Irshad, K. (2017). Molecular basis of hepatocellular carcinoma induced by hepatitis $\mathrm{C}$ virus infection. World J. Hepatol. 9, 1305-1314. https://doi. org/10.4254/wjh.v9.i36.1305.

Izumiya, Y., Lin, S.F., Ellison, T., Chen, L.Y., Izumiya, C., Luciw, P., and Kung, H.J. (2003). Kaposi's sarcomaassociated herpesvirus K-bZIP is a coregulator of K-Rta: physical association and promoter-dependent transcriptional repression. J. Virol. 77, 1441-1451.

Izumiya, Y., Ellison, T.J., Yeh, E.T., Jung, J.U., Luciw, P.A., and Kung, H.J. (2005). Kaposi's sarcoma-associated herpesvirus K-bZIP represses gene transcription via SUMO modification. J. Virol. 79, 9912-9925.

Izumiya, Y., Kobayashi, K., Kim, K.Y., Pochampalli, M., Izumiya, C., Shevchenko, B., Wang, D.H., Huerta, S.B., Martinez, A., Campbell, M., et al. (2013). Kaposi's sarcoma-associated herpesvirus K-Rta exhibits SUMOtargeting ubiquitin ligase (STUbL) like activity and is essential for viral reactivation. PLOS pathogens 9, e1003506. https://doi.org/10.1371/journal. ppat.1003506.

Jaiswal, N., John, R., Chand, V., and Nag, A. (2015). Oncogenic Human Papillomavirus 16E7 modulates SUMOylation of FoxM1b. Int. J. Biochem. Cell Biol. 58, 28-36. https://doi.org/10.1016/j.biocel.2014.11.002.

Jeang, K.T., Giam, C.Z., Majone, F., and Aboud, M. (2004). Life, death, and tax: role of HTLV-I oncoprotein in genetic instability and cellular transformation. J. Biol. Chem. 279, 31991-31994. https://doi.org/10.1074/ jbc.R400009200.

Jentsch, S., and Psakhye, I. (2013). Control of nuclear activities by substrate-selective and protein-group SUMOylation. Annu. Rev. Genet. 47, 167-186. https:// doi.org/10.1146/annurev-genet-111212-133453.

Johnson, E.S., and Gupta, A.A. (2001). An E3-like factor that promotes SUMO conjugation to the yeast septins. Cell 106, 735-744.

Kamitani, T., Nguyen, H.P., and Yeh, E.T. (1997). Preferential modification of nuclear proteins by a novel ubiquitinlike molecule. J. Biol. Chem. 272, 14001-14004.

Kerscher, O. (2007). SUMO junction-what's your function? New insights through SUMO-interacting motifs. EMBO Rep. 8, 550-555.

Kfoury, Y., Setterblad, N., El-Sabban, M., Zamborlini, A., Dassouki, Z., El Hajj, H., Hermine, O., Pique, C., de Thé, H., Saïb, A., et al. (2011). Tax ubiquitylation and SUMOylation control the dynamic shuttling of Tax and NEMO between Ubc9 nuclear bodies and the centrosome. Blood 117, 190-199. https://doi. org/10.1182/blood-2010-05-285742. 
Knipscheer, P., Flotho, A., Klug, H., Olsen, J.V., van Dijk, W.J., Fish, A., Johnson, E.S., Mann, M., Sixma, T.K., and Pichler, A. (2008). Ubc9 sumoylation regulates SUMO target discrimination. Mol. Cell 31, 371-382. https:// doi.org/10.1016/j.molcel.2008.05.022.

Kunz, K., Piller, T., and Müller, S. (2018). SUMO-specific proteases and isopeptidases of the SENP family at a glance. J. Cell. Sci. 131, jcs211904.

Lamsoul, I., Lodewick, J., Lebrun, S., Brasseur, R., Burny, A., Gaynor, R.B., and Bex, F. (2005). Exclusive ubiquitination and sumoylation on overlapping lysine residues mediate NF-kappaB activation by the human T-cell leukemia virus tax oncoprotein. Mol. Cell. Biol. 25, 10391-10406.

Lapenta, V., Chiurazzi, P., van der Spek, P., Pizzuti, A., Hanaoka, F., and Brahe, C. (1997). SMT3A, a human homologue of the S. cerevisiae SMT3 gene, maps to chromosome 21qter and defines a novel gene family. Genomics 40, 362-366.

Lee, H.S., Lim, Y.S., Park, E.M., Baek, S.H., and Hwang, S.B. (2014). SUMOylation of nonstructural 5A protein regulates hepatitis $C$ virus replication. J. Viral Hepat. 21, e108-17. https://doi.org/10.1111/jvh.12241.

Li, J., Callegari, S., and Masucci, M.G. (2017). The Epstein-Barr virus miR-BHRF1-1 targets RNF4 during productive infection to promote the accumulation of SUMO conjugates and the release of infectious virus. PLOS Pathog. 13, e1006338. https://doi.org/10.1371/ journal.ppat.1006338.

Li, R., Wang, L., Liao, G., Guzzo, C.M., Matunis, M.J., Zhu, H., and Hayward, S.D. (2012). SUMO binding by the Epstein-Barr virus protein kinase BGLF4 is crucial for BGLF4 function. J. Virol. 86, 5412-5421. https://doi. org/10.1128/JVI.00314-12.

Li, S.J., and Hochstrasser, M. (2000). The yeast ULP2 (SMT4) gene encodes a novel protease specific for the ubiquitin-like Smt3 protein. Mol. Cell. Biol. 20, 2367-2377.

Liang, Y.C., Lee, C.C., Yao, Y.L., Lai, C.C., Schmitz, M.L., and Yang, W.M. (2016). SUMO5, a novel poly-SUMO isoform, regulates PML nuclear bodies. Sci. Rep. 6, 26509. https://doi.org/10.1038/srep26509.

Lin, S.F., Robinson, D.R., Miller, G., and Kung, H.J. (1999). Kaposi's sarcoma-associated herpesvirus encodes a bZIP protein with homology to BZLF1 of Epstein-Barr virus. J. Virol. 73, 1909-1917.

Lin, X., Sun, R., Zhang, F., Gao, Y., Bin, L., and Lan, K. (2017). The latency-associated nuclear antigen of Kaposi's sarcoma-associated herpesvirus inhibits expression of SUMO/sentrin-specific peptidase 6 to facilitate establishment of latency. J. Virol. 91. https:// doi.org/10.1128/JVI.00806-17.

Liu, J., Sha, M., Wang, Q. Ma, Y., Geng, X., Gao, Y., Feng, L., Shen, Y., and Shen, Y. (2015). Small ubiquitin-related modifier 2/3 interacts with p65 and stabilizes it in the cytoplasm in $\mathrm{HBV}$-associated hepatocellular carcinoma. BMC Cancer 15, 675. https://doi.org/10.1186/ s12885-015-1665-3.

Lowrey, A.J., Cramblet, W., and Bentz, G.L. (2017). Viral manipulation of the cellular sumoylation machinery. Cell Commun. Signal 15, 27. https://doi.org/10.1186/ s12964-017-0183-0.
Mahajan, R., Delphin, C., Guan, T., Gerace, L., and Melchior, F. (1997). A small ubiquitin-related polypeptide involved in targeting RanGAP1 to nuclear pore complex protein RanBP2. Cell 88, 97-107.

Maison, C., Romeo, K., Bailly, D., Dubarry, M., Quivy, J.P., and Almouzni, G. (2012). The SUMO protease SENP7 is a critical component to ensure HP1 enrichment at pericentric heterochromatin. Nat. Struct. Mol. Biol. 19, 458-460. https://doi.org/10.1038/nsmb.2244.

Mannen, H., Tseng, H.M., Cho, C.L., and Li, S.S. (1996). Cloning and expression of human homolog HSMT3 to yeast SMT3 suppressor of MIF2 mutations in a centromere protein gene. Biochem. Biophys. Res. Commun. 222, 178-180.

Marusic, M.B., Mencin, N., Licen, M., Banks, L., and Grm, H.S. (2010). Modification of human papillomavirus minor capsid protein L2 by sumoylation. J. Virol. 84, 11585-11589. https://doi.org/10.1128/JVI.01269-10.

Mattoscio, D., and Chiocca, S. (2015). SUMO pathway components as possible cancer biomarkers. Future Oncol. 11, 1599-1610. https://doi.org/10.2217/ fon.15.41.

Mattoscio, D., Segré, C.V., and Chiocca, S. (2013). Viral manipulation of cellular protein conjugation pathways: The SUMO lesson. World J. Virol. 2, 79-90. https://doi. org/10.5501/wjv.v2.i2.79.

Mattoscio, D., Casadio, C., Fumagalli, M., Sideri, M., and Chiocca, S. (2015). The SUMO conjugating enzyme UBC9 as a biomarker for cervical HPV infections. Ecancermedicalscience 9, 534. https://doi. org/10.3332/ecancer.2015.534.

Mattoscio, D., Casadio, C., Miccolo, C., Maffini, F., Raimondi, A., Tacchetti, C., Gheit, T., Tagliabue, M., Galimberti, V.E., De Lorenzi, F., et al. (2017). Autophagy regulates UBC9 levels during viral-mediated tumorigenesis. PLOS Pathog. 13, e1006262. https:// doi.org/10.1371/journal.ppat.1006262.

Mattoscio, D., Medda, A., and Chiocca, S. (2018). Human Papilloma Virus and Autophagy. International journal of molecular sciences 19. doi: 10.3390/ijms19061775.

Matunis, M.J., Coutavas, E., and Blobel, G. (1996). A novel ubiquitin-like modification modulates the partitioning of the Ran-GTPase-activating protein RanGAP1 between the cytosol and the nuclear pore complex. J. Cell Biol. 135, 1457-1470.

McBride, A.A. (2013). The papillomavirus E2 proteins. Virology 445, 57-79. https://doi.org/10.1016/j. virol.2013.06.006.

Mesri, E.A., Feitelson, M.A., and Munger, K. (2014). Human viral oncogenesis: a cancer hallmarks analysis. Cell Host Microbe 15, 266-282. https://doi.org/10.1016/j. chom.2014.02.011.

Mohideen, F., Capili, A.D., Bilimoria, P.M., Yamada, T., Bonni, A., and Lima, C.D. (2009). A molecular basis for phosphorylation-dependent SUMO conjugation by the E2 UBC9. Nat. Struct. Mol. Biol. 16, 945-952. https:// doi.org/10.1038/nsmb.1648.

Moody, C.A., and Laimins, L.A. (2010). Human papillomavirus oncoproteins: pathways to transformation. Nat. Rev. Cancer 10, 550-560. https:// doi.org/10.1038/nrc2886.

Munk, S., Sigurðsson, J.O., Xiao, Z., Batth, T.S., Franciosa, G., von Stechow, L., Lopez-Contreras, A.J., Vertegaal, 
A.C.O., and Olsen, J.V. (2017). Proteomics reveals global regulation of protein SUMOylation by ATM and ATR kinases during replication stress. Cell Rep. 21, 546-558.

Murata, T., Hotta, N., Toyama, S., Nakayama, S., Chiba, S., Isomura, H., Ohshima, T., Kanda, T., and Tsurumi, T. (2010). Transcriptional repression by sumoylation of Epstein-Barr virus BZLF1 protein correlates with association of histone deacetylase. J. Biol. Chem. 285, 23925-23935. https://doi.org/10.1074/jbc. M109.095356.

Myatt, S.S., and Lam, E.W. (2007). The emerging roles of forkhead box (Fox) proteins in cancer. Nat. Rev. Cancer 7, 847-859.

Myatt, S.S., Kongsema, M., Man, C.W., Kelly, D.J., Gomes, A.R., Khongkow, P., Karunarathna, U., Zona, S., Langer, J.K., Dunsby, C.W., et al. (2014). SUMOylation inhibits FOXM1 activity and delays mitotic transition. Oncogene 33, 4316-4329. https://doi.org/10.1038/ onc.2013.546.

Nasr, R., Chiari, E., El-Sabban, M., Mahieux, R., Kfoury, Y., Abdulhay, M., Yazbeck, V., Hermine, O., de Thé, H., Pique, C., et al. (2006). Tax ubiquitylation and sumoylation control critical cytoplasmic and nuclear steps of NF-kappaB activation. Blood 107, 4021-4029.

Neumann, F., Czech-Sioli, M., Dobner, T., Grundhoff, A., Schreiner, S., and Fischer, N. (2016). Replication of Merkel cell polyomavirus induces reorganization of promyelocytic leukemia nuclear bodies. J. Gen. Virol. 97, 2926-2938. https://doi.org/10.1099/jgv.0.000593.

Owerbach, D., McKay, E.M., Yeh, E.T., Gabbay, K.H., and Bohren, K.M. (2005). A proline-90 residue unique to SUMO-4 prevents maturation and sumoylation. Biochem. Biophys. Res. Commun. 337, 517-520.

Perry, J.J., Tainer, J.A., and Boddy, M.N. (2008). A SIMultaneous role for SUMO and ubiquitin. Trends Biochem. Sci. 33, 201-208. https://doi.org/10.1016/j. tibs.2008.02.001.

Plummer, M., de Martel, C., Vignat, J., Ferlay, J., Bray, F., and Franceschi, S. (2016). Global burden of cancers attributable to infections in 2012: a synthetic analysis. Lancet Glob. Health 4, e609-16. https://doi. org/10.1016/S2214-109X(16)30143-7.

Poiesz, B.J., Ruscetti, F.W., Gazdar, A.F., Bunn, P.A., Minna, J.D., and Gallo, R.C. (1980). Detection and isolation of type $\mathrm{C}$ retrovirus particles from fresh and cultured lymphocytes of a patient with cutaneous T-cell lymphoma. Proc. Natl. Acad. Sci. U.S.A. 77, 7415-7419.

Pozzebon, M.E., Varadaraj, A., Mattoscio, D., Jaffray, E.G., Miccolo, C., Galimberti, V., Tommasino, M., Hay, R.T., and Chiocca, S. (2013). BC-box protein domain-related mechanism for VHL protein degradation. Proc. Natl. Acad. Sci. U.S.A. 110, 18168-18173. https://doi. org/10.1073/pnas.1311382110.

Psakhye, I., and Jentsch, S. (2012). Protein group modification and synergy in the SUMO pathway as exemplified in DNA repair. Cell 151, 807-820.

Reverter, D., and Lima, C.D. (2006). Structural basis for SENP2 protease interactions with SUMO precursors and conjugated substrates. Nat. Struct. Mol. Biol. 13, 1060-1068.

Rodriguez, M.S., Dargemont, C., and Hay, R.T. (2001). SUMO-1 conjugation in vivo requires both a consensus modification motif and nuclear targeting. J. Biol. Chem.
276, 12654-12659. https://doi.org/10.1074/jbc. M009476200.

Ropero, S., and Esteller, M. (2007). The role of histone deacetylases (HDACs) in human cancer. Mol. Oncol. 1, 19-25.https://doi.org/10.1016/j.molonc.2007.01.001.

Sachdev, S., Bruhn, L., Sieber, H., Pichler, A., Melchior, F., and Grosschedl, R. (2001). PIASy, a nuclear matrixassociated SUMO E3 ligase, represses LEF1 activity by sequestration into nuclear bodies. Genes Dev. 15, 3088-3103.

Seeler, J.S., and Dejean, A. (2017). SUMO and the robustness of cancer. Nat. Rev. Cancer 17, 184-197. https://doi.org/10.1038/nrc.2016.143.

Sengupta, I., Das, D., Singh, S.P., Chakravarty, R., and Das, C. (2017). Host transcription factor Speckled $110 \mathrm{kDa}$ (Sp110), a nuclear body protein, is hijacked by hepatitis $\mathrm{B}$ virus protein $\mathrm{X}$ for viral persistence. J. Biol. Chem. 292, 20379-20393. https://doi.org/10.1074/jbc. M117.796839.

Shen, L., Tatham, M.H., Dong, C., Zagórska, A., Naismith, J.H., and Hay, R.T. (2006a). SUMO protease SENP1 induces isomerization of the scissile peptide bond. Nat. Struct. Mol. Biol. 13, 1069-1077.

Shen, T.H., Lin, H.K., Scaglioni, P.P., Yung, T.M., and Pandolfi, P.P. (2006b). The mechanisms of PML-nuclear body formation. Mol. Cell 24, 331-339.

Shen, Z., Pardington-Purtymun, P.E., Comeaux, J.C., Moyzis, R.K., and Chen, D.J. (1996). UBL1, a human ubiquitin-like protein associating with human RAD51/ RAD52 proteins. Genomics 36, 271-279.

Shuda, M., Kwun, H.J., Feng, H., Chang, Y., and Moore, P.S. (2011). Human Merkel cell polyomavirus small $\mathrm{T}$ antigen is an oncoprotein targeting the 4E-BP1 translation regulator. J. Clin. Invest. 121, 3623-3634. https://doi.org/10.1172/JCI46323.

Spurgeon, M.E., and Lambert, P.F. (2013). Merkel cell polyomavirus: a newly discovered human virus with oncogenic potential. Virology 435, 118-130. https:// doi.org/10.1016/j.virol.2012.09.029.

Sriramachandran, A.M., and Dohmen, R.J. (2014). SUMOtargeted ubiquitin ligases. Biochim. Biophys. Acta 1843, 75-85. https://doi.org/10.1016/j.bbamcr.2013.08.022.

Su, Y.F., Yang, T., Huang, H., Liu, L.F., and Hwang, J. (2012). Phosphorylation of Ubc9 by Cdk1 enhances SUMOylation activity. PLOS ONE 7, e34250. https:// doi.org/10.1371/journal.pone.0034250.

Sun, H., Leverson, J.D., and Hunter, T. (2007). Conserved function of RNF4 family proteins in eukaryotes: targeting a ubiquitin ligase to SUMOylated proteins. EMBO J. 26, 4102-4112.

Sun, R., Liang, D., Gao, Y., and Lan, K. (2014). Kaposi's sarcoma-associated herpesvirus-encoded LANA interacts with host KAP1 to facilitate establishment of viral latency. J. Virol. 88, 7331-7344. https://doi. org/10.1128/JVI.00596-14.

Tatham, M.H., Kim, S., Jaffray, E., Song, J., Chen, Y., and Hay, R.T. (2005). Unique binding interactions among Ubc9, SUMO and RanBP2 reveal a mechanism for SUMO paralog selection. Nat. Struct. Mol. Biol. 12, 67-74.

Thompson, M.P., and Kurzrock, R. (2004). Epstein-Barr virus and cancer. Clin. Cancer Res. 10, 803-821.

Tomasi, M.L., and Ramani, K. (2018). SUMOylation and phosphorylation cross-talk in hepatocellular carcinoma. 
Transl. Gastroenterol. Hepatol. 3, 20. https://doi. org/10.21037/tgh.2018.04.04.

Tommasino, M. (2014). The human papillomavirus family and its role in carcinogenesis. Semin. Cancer Biol. 26, 13-21. https://doi.org/10.1016/j. semcancer.2013.11.002.

Tong, H., Hateboer, G., Perrakis, A., Bernards, R., and Sixma, T.K. (1997). Crystal structure of murine/human Ubc9 provides insight into the variability of the ubiquitinconjugating system. J. Biol. Chem. 272, 21381-21387.

Tsurumi, T., Fujita, M., and Kudoh, A. (2005). Latent and lytic Epstein-Barr virus replication strategies. Rev. Med. Virol. 15, 3-15. https://doi.org/10.1002/rmv.441.

Uppal, T., Banerjee, S., Sun, Z., Verma, S.C., and Robertson, E.S. (2014). KSHV LANA - the master regulator of KSHV latency. Viruses 6, 4961-4998. https://doi. org/10.3390/v6124961.

van der Horst, A., and Burgering, B.M. (2007). Stressing the role of FoxO proteins in lifespan and disease. Nat. Rev. Mol. Cell Biol. 8, 440-450.

Wang, C.Y., Podolsky, R., and She, J.X. (2006). Genetic and functional evidence supporting SUMO4 as a type 1 diabetes susceptibility gene. Ann. N. Y. Acad. Sci. 1079, 257-267.

Wang, J., Guo, Y., Wang, X., Zhao, R., and Wang, Y. (2017). Modulation of global SUMOylation by Kaposi's sarcoma-associated herpesvirus and its effects on viral gene expression. J. Med. Virol. 89, 2011-2019. https:// doi.org/10.1002/jmv.24882.

Wilkinson, K.A., and Henley, J.M. (2010). Mechanisms, regulation and consequences of protein SUMOylation. Biochem. J. 428, 133-145. https://doi.org/10.1042/ BJ20100158.

Wilson, V.G. (2009). SUMO regulation of cellular processes (Springer, Dordrecht).

Wilson, V.G. (2017). Viral interplay with the host sumoylation system. Adv. Exp. Med. Biol. 963, 359-388. https://doi.org/10.1007/978-3-319-50044-7_21.

Woodman, C.B., Collins, S.I., and Young, L.S. (2007). The natural history of cervical HPV infection: unresolved issues. Nat. Rev. Cancer 7, 11-22.
Wu, Y.C., Deyrieux, A.F., and Wilson, V.G. (2007). Papillomaviruses and the host SUMOylation system. Biochem. Soc. Trans. 35, 1433-1435. https://doi. org/10.1042/BST0351433.

Wu, Y.C., Roark, A.A., Bian, X.L., and Wilson, V.G. (2008). Modification of papillomavirus E2 proteins by the small ubiquitin-like modifier family members (SUMOs). Virology 378, 329-338. https://doi.org/10.1016/j. virol.2008.06.008.

Wu, Y.C., Bian, X.L., Heaton, P.R., Deyrieux, A.F., and Wilson, V.G. (2009). Host cell sumoylation level influences papillomavirus E2 protein stability. Virology 387, 176-183. https://doi.org/10.1016/j. virol.2009.02.002.

Xie, Y. (2017). Hepatitis B virus-associated hepatocellular carcinoma. Adv. Exp. Med. Biol. 1018, 11-21. https:// doi.org/10.1007/978-981-10-5765-6 2.

Yang, S.H., Galanis, A., Witty, J., and Sharrocks, A.D. (2006). An extended consensus motif enhances the specificity of substrate modification by SUMO. EMBO J. 25, 5083-5093.

Yang, W.S., Hsu, H.W., Campbell, M., Cheng, C.Y., and Chang, P.C. (2015). K-bZIP mediated SUMO-2/3 specific modification on the KSHV genome negatively regulates lytic gene expression and viral reactivation. PLOS Pathog. 11, e1005051. https://doi.org/10.1371/ journal.ppat.1005051.

Yang, Y., He, Y., Wang, X., Liang, Z., He, G., Zhang, P., Zhu, H., Xu, N., and Liang, S. (2017). Protein SUMOylation modification and its associations with disease. Open Biol. 7, 170167.

Yang, Y.C., Feng, T.H., Chen, T.Y., Huang, H.H., Hung, C.C., Liu, S.T., and Chang, L.K. (2015). RanBPM regulates Zta-mediated transcriptional activity in Epstein-Barr virus. J. Gen. Virol. 96, 2336-2348. https://doi. org/10.1099/vir.0.000157.

Zhao, X. (2018). SUMO-mediated regulation of nuclear functions and signaling processes. Mol. Cell 71, 409418.

zur Hausen, H. (2009). Papillomaviruses in the causation of human cancers - a brief historical account. Virology 384, 260-265. https://doi.org/10.1016/j.virol.2008.11.046 